\title{
Ultraviolet Observations of Interstellar Molecules
}

\author{
Theodore P. Snow $\dagger$ \\ University of Colorado, Boulder, CO, USA \\ email: tsnow@casa.colorado.edu
}

\begin{abstract}
The benefits of placing ultraviolet (UV) spectrographs in space were first pointed out by Spitzer (1946) and Spitzer \& Zabriskie (1959), with much of the emphasis on absorptionline measurements of interstellar atoms and molecules. The first UV observations of the diffuse interstellar medium took place in the early 1970s, using sounding rockets, which were followed by a series of orbital instruments starting in the later 1970s and extending almost to the present time. Many of these observations have provided information on the chemistry of the diffuse ISM, and will be emphasized here. The most abundant molecule in space, $\mathrm{H}_{2}$, is not readily observed in other wavelength bands and was first detected by a UV sounding rocket experiment (Carruthers 1970). Thereafter a series of orbital instruments, starting with the Copernicus satellite in 1972 and culminating with the launch of FUSE in 1999, carried out broad surveys of molecular hydrogen in a variety of ISM environments (e.g., Spitzer et al. 1973; Snow et al. 2000; Shull et al. 2000; see also the review by Shull \& Beckwith 1982). The UV observations of electronic transitions of $\mathrm{H}_{2}$, including lines arising from excited rotational levels of the ground state, have provided a wealth of information on not only the chemistry of the diffuse ISM, but also the composition and physical conditions. The second most abundant molecule in the diffuse ISM, $\mathrm{CO}$, also has numerous electronic transitions in the UV, and has been widely observed by many different instruments, most usefully the Hubble Space Telescope (HST) (e.g., Lambert et al. 1994; Sheffer et al. 2002). Valuable information on diffuse ISM chemistry, isotopic composition, and physical conditions has been provided by these observations. A few additional molecules, all of them diatomics such as $\mathrm{OH}$ and $\mathrm{N}_{2}$, have been detected through UV absorption-line observations, and many others have been sought unsuccessfully. The Cosmic Origins Spectrograph, expected to be installed aboard the HST in 2007, will have enhanced UV sensitivity and will provide information on molecular abundances in denser clouds than previously studied. One of the high priorities will be the search for electronic transitions of complex organic molecules such as PAHs.
\end{abstract}

Keywords. molecular data - molecular processes — techniques: spectroscopic — ISM: abundances — ISM: atoms — ISM: molecules — ultraviolet: ISM

\section{Introduction}

For reasons described in detail below, ultraviolet studies of molecules in the interstellar medium (ISM) are restricted to diffuse clouds. Therefore UV measurements do not apply to the dense molecular clouds where star formation takes place. Nevertheless, because the chemistry in diffuse clouds is simpler yet still applicable to dense clouds, it is very useful to apply UV spectroscopy to the study of molecules in interstellar clouds. This paper therefore provides a brief review of what has been learned from ultraviolet observations of interstellar molecules in diffuse clouds.

In the diffuse interstellar medium, collisions are rare and most atoms, ions, and molecules are in their ground electronic state. For molecules, only very low-lying rotational states of the ground vibrational level are likely to be populated, except in regions where

$\dagger$ Present address: CASA - University of Colorado, 389 UCB, Boulder, CO 80309-0389 
there is a local excitation source such as a strong radiation field. For the majority of these species transitions from the ground electronic state to higher levels require sufficient energy to fall in the ultraviolet portion of the spectrum. Therefore, perhaps a bit ironically, observations of absorption lines formed by cold interstellar atoms and molecules require the high-energy photons of ultraviolet radiation.

Despite the richness of infrared and mm-wave radio observations of interstellar molecules, there are a few species that are best observed through their electronic transitions in the UV. These include the most abundant interstellar molecule of all, $\mathrm{H}_{2}$, as well as its isotopologue, HD. In addition the second most abundant molecule in space, CO, can be observed in diffuse clouds through its UV electronic transitions, while IR and radio methods are superior for denser clouds. Many other species have electronic transitions in the UV, including both $\mathrm{OH}$ and $\mathrm{H}_{2} \mathrm{O}$ as well as various large organic species suspected of being common in the diffuse ISM but not yet identified.

Ultraviolet radiation does not penetrate dense interstellar material, so as noted above, UV observations of interstellar molecules are restricted to diffuse clouds. Here we refer to diffuse atomic clouds and diffuse molecular clouds, which are defined on the basis of their molecular hydrogen content. Clouds containing little or no $\mathrm{H}_{2}$ are identified as diffuse atomic clouds, while those containing a significant molecular fraction are diffuse molecular clouds. The preceding paper by B. J. McCall contains further comments on this distinction between cloud types, as does the review by Snow \& McCall (2006).

Despite being limited to diffuse atomic and molecular clouds, UV observations of interstellar molecules have made significant contributions to our understanding of astrochemistry. Important experiments and missions in the past include the sounding rockets used to make the initial discoveries of molecular hydrogen (Carruthers 1970) and the UV transitions of CO (Smith \& Stecher 1971); the Copernicus observatory, which operated in the 1970s and carried out pioneering studies of interstellar atomic and molecular abundances (particularly $\mathrm{H}_{2}$ ); the International Ultraviolet Explorer (IUE), which was especially useful in measuring dust extinction curves; both the GHRS and STIS instruments on the Hubble Space Telescope (HST); and the Far Ultraviolet Spectroscope Explorer (FUSE), which at this writing was the only UV spectroscopic instrument still operating.

No new UV missions are being planned, so the future for UV astronomy is grim, though we can hope that the Cosmic Origins Spectrograph (Green 2000), already built and calibrated and ready to go, will eventually be deployed on the $H S T$.

\section{Technical Challenges to UV Spectroscopy}

The primary challenge facing observers of UV interstellar spectra is brought about by the Earth's atmosphere, which refuses to allow UV photons to enter from space. Thus UV telescopes must be launched into space, an expensive prospect.

A second challenge, more readily surmounted, is to design and build telescope optics that will be reflective at UV wavelengths. Most materials become absorptive in the UV, more so with decreasing wavelength. But great success has been achieved with aluminum mirrors that are overcoated with substances such as magnesium fluoride $\left(\mathrm{MgF}_{2}\right)$ or lithium fluoride $(\mathrm{LiF})$, which transmit UV radiation while protecting the aluminum from oxidation, the chief cause of reflectivity loss. An alternative far-UV reflective surface is silicon carbide ( $\mathrm{SiC}$ ), which has been used successfully as well, for example in the FUSE telescope optics.

An additional obstacle to UV observations of interstellar lines, one which cannot be avoided, is that the interstellar medium itself blocks out UV radiation, in proportion to distance or column density. The most drastic effect is caused by interstellar dust, which 
absorbs and scatters photons with increasing efficiency toward short UV wavelengths. The resulting extinction curve rises from the infrared through to the far-UV, reaching levels below $1200 \AA$ as high as 10 or 15 times the color excess $E_{B-V}$, which translates to UV attenuation factors of 10,000 to 100,000 in comparison with unreddened stars. Unfortunately, the diffuse clouds with the highest molecular abundances are also the ones with the steepest and most obstructive UV dust extinction curves.

Another difficulty in observing interstellar molecules at UV wavelengths is caused, ironically, by interstellar molecules. In reddened lines of sight $\mathrm{H}_{2}$ bands wipe out large segments of the spectrum below $1110 \AA$, preventing observations of interstellar lines due to other species. The saturated (often damped) lines arising from the $J=0$ and $J=1$ rotational levels of the ground electronic and vibrational states of $\mathrm{H}_{2}$ are strong enough to obliterate several $\AA$ at the location of each of the many Lyman and Werner bands that are spaced regularly from $1108 \AA$ all the way through to the Lyman limit at $912 \AA$.

Finally, yet another complication for observations of UV interstellar lines is that the hot stars that are used as background sources themselves have complex spectra. Whereas $\mathrm{O}$ and $\mathrm{B}$ stars have smooth continua at visible wavelengths, in the UV their spectra are crowded with lines, adding to the difficulty of detecting and analyzing interstellar features. The problem is ameliorated by selecting stars with high rotational velocities, whose photospheric lines are much broader than most interstellar lines-but even so, the continuum against which the interstellar lines are measured is rarely smooth and level.

Despite the many difficulties, UV spectroscopy has proven to be an extremely valuable tool for studies of the diffuse ISM, including observations of molecular species.

\section{Aside: What We Can Learn about the Atomic Gas from UV Spectroscopy}

In order to place molecular observations into context, for example in order to test observations against chemical models, it is necessary to have a general picture of what we can learn from UV observations of atomic lines formed in the same diffuse clouds where the observed molecules reside. Several useful reviews of UV atomic line observations have been published, including those by Jenkins \& Spitzer (1975), Savage \& Sembach (1996); and Snow (1997).

As noted in the Introduction, many species, both atomic and molecular, have their ground state absorption transitions in the ultraviolet. Most of the common elements, from hydrogen through the iron peak, can be observed in the UV, often in their dominant ionization state. In the diffuse ISM the ambient interstellar radiation field, which approximates a black body having a temperature of around 10,000 K, ensures that most species whose ionization potentials are below the $13.6 \mathrm{eV}$ Lyman limit of hydrogen are singly ionized. Atomic hydrogen permeating the diffuse ISM obliterates all photons with energies immediately above $13.6 \mathrm{eV}$ (i.e., wavelengths just below $912 \AA$ ). Thus species such as nitrogen and oxygen, whose ionization potentials are near or above $13.6 \mathrm{eV}$, remain mostly in the neutral atomic form while most other common elements through the iron peak are found in the singly ionized state (with the notable exception of helium, which is simply unobservable in diffuse clouds except for the very local ISM where extreme UV spectra can be obtained).

Observations of the UV absorption lines of common elements lead to derivations of their relative abundances, and in turn to estimates of elemental depletions; i.e., the factors by which the elements in the diffuse ISM are underabundant relative to a reference standard, which is often taken to be the solar composition. Most elements are depleted to some extent (relative to hydrogen), ranging from less than a factor of two for volatiles such 
as $\mathrm{C}, \mathrm{N}$, and $\mathrm{O}$ to factors from $10^{2}$ to $10^{4}$ for some of the refractory elements. What is missing from the gas is assumed to be present in dust grains, so depletion measures are an indirect means of determining the composition of the dust.

Of greater relevance for understanding the chemistry of diffuse clouds is what the atomic line observations can tell us about the physical conditions in diffuse clouds, because factors such as the volume density of atoms, the electron density, and the radiation field intensity, all of which can be derived from data on atomic lines, are important in assessing the chemical environment. The volume density of atoms (or molecules) can be estimated from the analysis of fine-structure collisional excitation in neutral atoms such as atomic carbon (CI) and ionized carbon, nitrogen, and silicon (C II, N II, and Si II) (see Bahcall \& Wolf 1968 or Jenkins \& Shaya 1981 for details). The electron density can be deduced from the ionization ratios of elements where both the neutral and cationic forms are observed, such as carbon, magnesium, iron, and others (see Morton 1975 for a summary of this analysis for the line of sight toward $\zeta \mathrm{Oph}$ ). The radiation field intensity is best derived from the analysis of rotational excitation in molecules such as $\mathrm{H}_{2}$ and $\mathrm{C}_{2}$ whose excitation is dominated by UV pumping (this will be described in a later section).

Finally, high-resolution spectra of atomic interstellar line profiles can reveal the velocity distribution along a line of sight, thereby constraining the interpretation of observed molecular lines. Most such observations to date have been made at visible wavelengths where spectrographs are available with resolving powers $(\lambda / \Delta \lambda)$ as high as $10^{6}$. Application of velocity structure information to lower-resolution UV spectra requires line profile modeling, but has proven very useful in constraining and interpreting interstellar abundances (ideally we would have an ultraviolet spectrograph with ultra-high resolving power, but as noted above, the prospects for this any time soon are poor).

\section{Interstellar Molecules Detected through UV Observations}

A number of molecules have been detected through UV absorption lines (Table 1). By far the two most abundant species, not only for UV measurements but in dense clouds as well, are molecular hydrogen and carbon monoxide. But a few other species have been detected as well, and are important for constraining models of diffuse cloud chemistry.

Table 1. Molecules detected through UV observations

\begin{tabular}{cccl}
\hline Species & $\begin{array}{c}\text { log column density } \\
\text { (for } \zeta \text { Oph) }\end{array}$ & $\begin{array}{c}\text { Abundance } \\
\text { relative to } \mathrm{H}\end{array}$ & Reference \\
\hline $\mathrm{H}_{2}$ & 20.62 & 0.31 & Morton 1975 \\
$\mathrm{H}_{2}(v=3)$ & 11.81 & $4.8 \times 10^{-10}$ & Federman et al. 1995 \\
$\mathrm{HD}$ & 14.80 & $6.8 \times 10^{-7}$ & Lacour et al. 1995 \\
$\mathrm{CO}$ & 15.40 & $1.9 \times 10^{-6}$ & Lambert et al. 1994 \\
${ }^{13} \mathrm{CO}$ & 13.17 & $1.1 \times 10^{-8}$ & Lambert et al. 1994 \\
$\mathrm{OH}$ & 13.70 & $3.7 \times 10^{-8}$ & Crutcher \& Watson 1976 \\
$\mathrm{HCl}$ & 11.43 & $2.0 \times 10^{-10}$ & Federman et al. 1995 \\
$\mathrm{~N}_{2}{ }^{\dagger}$ & 13.66 & $1.6 \times 10^{-7}$ & Knauth et al. 2004 \\
\hline
\end{tabular}

$\dagger$ The values for $\mathrm{N}_{2}$ is not for $\zeta$ Oph but instead for the star HD 124314, which has a total visual extinction of about $1.5 \mathrm{mag}$ as opposed to about $1.0 \mathrm{mag}$ for $\zeta$ Oph. 


\subsection{Ultraviolet Observations of $\mathrm{H}_{2}$ and $\mathrm{CO}$}

\subsubsection{Molecular Hydrogen}

$\mathrm{H}_{2}$ is a homonuclear diatomic species having no dipole moment and hence no allowed rotational or vibrational transitions. Therefore, with the exception of forbidden quadrupole emission seen near $2.2 \mu \mathrm{m}$ in the near infrared in radiatively excited nebulae, $\mathrm{H}_{2}$ is virtually unobservable in the diffuse ISM except through its allowed electronic transitions in the ultraviolet. However, $\mathrm{H}_{2}$ was always expected to be the most abundant molecule in space, leading to the development of spectrographs specifically aimed at observing this species. The initial detection of interstellar $\mathrm{H}_{2}$ was achieved by Carruthers (1970) with a small rocket-borne spectrograph, and the first extensive surveys of molecular hydrogen in the ISM were carried out by the Copernicus observatory (Rogerson et al. 1973; Spitzer et al. 1973; Spitzer, Cochran, \& Hirshfeld 1974; Savage et al. 1977; Jenkins \& Spitzer 1975; Shull \& Beckwith 1982). More recently interstellar $\mathrm{H}_{2}$ absorption has been recorded by the IMAPS and ORFEUS Space Shuttle payloads (Jenkins et al. 1996; Hurwitz et al. 1998) and extensively by the Far-Ultraviolet Spectroscopic Explorer (FUSE) mission (Moos et al. 2000). The FUSE observations in particular have extended observations of molecular hydrogen to more reddened galactic lines of sight than previously explored; have provided sensitive surveys of $\mathrm{H}_{2}$ throughout the Milky Way's disk and halo; and have provided the first surveys of interstellar $\mathrm{H}_{2}$ in the Magellanic Clouds.

The essential results of the UV surveys of interstellar $\mathrm{H}_{2}$ are: (a) molecular hydrogen is EVERYWHERE in space, including lines of sight with very low reddening; (b) in diffuse clouds the fraction of hydrogen atoms in the form of $\mathrm{H}_{2}$ ranges from very small values ( 0.01 or less) to as large as 0.8 ; (c) there is a sharp transition from very low to very significant molecular fraction that occurs at a total hydrogen column density of about $5 \times 10^{19} \mathrm{~cm}^{-2}$ (corresponding to $E_{B-V} \approx 0.08 \mathrm{mag}$ ); (d) the relative populations of $\mathrm{H}_{2}$ molecules in rotationally excited states of the ground electronic state typically fit two different excitation temperatures; (e) vibrationally excited $\mathrm{H}_{2}$ is rarely seen in absorption in diffuse clouds; and (f) $\mathrm{H}_{2}$ in the Magellanic Clouds shows a reduced molecular fraction per reddening as compared with the Galaxy. Each of these points is expanded below.

On the ubiquity of interstellar $\mathrm{H}_{2}$ : Early data from Copernicus observations indicated that even for lines of sight with interstellar dust color excess $E_{B-V} \leqslant 0.1$ molecular hydrogen absorption could be detected. Now FUSE observations, notably the diffuse sightline surveys of Shull et al. (2001; 2002) have confirmed that $\mathrm{H}_{2}$ is nearly always detectable, with a range of column densities from about $10^{14} \mathrm{~cm}^{-2}$ to over $10^{20} \mathrm{~cm}^{-2}$. Few or no lines of sight have been found which do not contain detectable molecular hydrogen absorption.

The molecular fraction: Molecular hydrogen represents only a small fraction of all hydrogen atoms in lightly-reddened lines of sight (below about $E_{B-V}=0.1$ or so). For higher reddenings the molecular fraction rises, to a maximum value (to date) of roughly 0.8. No sightlines have been found with 100 percent of the hydrogen in molecular form, though it is possible that relatively dense clouds in some cases may approach this value (the difficulty is that every line of sight contains at least some diffuse material where much of the hydrogen is in atomic form; we cannot isolate specific dense clouds to ascertain their molecular fractions individually). One current issue is whether translucent clouds (as defined by van Dishoeck \& Black 1989, in which the hydrogen should be fully molecular while there is still sufficient penetration by UV photons to affect the chemistry) actually exist. While to date FUSE observations have found no molecular fraction of unity, a few sightlines have been identified which may contain translucent clouds where all the 
hydrogen is molecular-but as yet this has been difficult to establish due to the mix of clouds and environments along every line of sight.

The transition at $N_{H} \approx 5 \times 10^{19} \mathrm{~cm}^{-2}$ : There is a very significant break in the molecular fraction when total column densities exceed about $10^{19} \mathrm{~cm}^{-2}$, as shown first by Spitzer et al. (1973) and Savage et al. (1977) on the basis of Copernicus data and then verified by the various FUSE surveys. The explanation for this transition from low to high molecular fraction is straightforward: When the column density of $\mathrm{H}_{2}$ reaches about $10^{19} \mathrm{~cm}^{-2}$, the strong electronic transitions in the far-UV Lyman and Werner bands become saturated, so that the molecules in the outer portion of a cloud shield those in the interior from the UV photons that would otherwise destroy them.

Rotational excitation of $\mathrm{H}_{2}$ in diffuse clouds: Typically most of the observed $\mathrm{H}_{2}$ in diffuse clouds is in the first two rotational states $(J=0,1)$ of the ground vibrational level of the ground electronic state. But observations reveal in addition substantial populations in higher rotational states, up to $J=6$ or so in diffuse clouds (Spitzer et al. 1973; Spitzer \& Cochran 1973; Spitzer, Cochran, \& Hirshfeld 1974). In clouds sufficiently dense for the strong lines of $\mathrm{H}_{2}$ to be self-shielded, radiative processes do not affect the $J=0$ and $J=1$ levels, so that local chemical reactions govern their relative populations. As a result the ratio $N(J=1) / N(J=0)$ is a local thermometer, indicating the kinetic temperature of the gas. In diffuse clouds kinetic temperatures range from 50 to $150 \mathrm{~K}$. The higher- $J$ lines are believed to be populated by radiative pumping, as first pointed out by Black \& Dalgarno (1973) and then explored further by Jura (1975a,b), who developed useful models for deriving both the radiation field intensity and the local density for the optically thin and thick cases. In this process the high- $J$ levels are populated due to UV absorption resulting in excitation to an excited electronic state, from which the molecules either dissociate (a small fraction of the time) or cascade back down to the ground electronic state through a series of vibrational and rotational levels. Thus in equilibrium, in the presence of a strong UV radiation field, there is a constant population of $\mathrm{H}_{2}$ molecules in high- $J$ rotational states.

Vibrationally excited $\mathrm{H}_{2}$ in diffuse clouds: In rare cases absorption lines arising from excited vibrational states are seen, as in $\zeta$ Oph (Federman et al. 1995), HD 37903 (Meyer et al. 2001), and HD 34078 (Boisse et al. 2005). The vibrational states show up only in $\mathrm{H}$ II regions close to a hot exciting star-and are accompanied by much higher rotational states (up to $J=11$ in the case of HD 34078) than normally seen in diffuse clouds. Also, in $\mathrm{H}$ II regions and photon-dominated regions (PDRs), forbidden quadrupole emission from vibrationally and rotationally excited $\mathrm{H}_{2}$ is commonly seen near $2.2 \mu \mathrm{m}$ in the infrared, as is ultraviolet fluorescence emission in some cases (e.g., IC 63, a small reflection nebula excited by the star $\gamma$ Cas; Witt et al. 1989; Hurwitz 1998).

Molecular hydrogen in the Magellanic Clouds: Using FUSE data, Tumlinson et al. (2002) amassed a survey of some 70 lines of sight toward hot stars in both Magellanic Clouds, finding that the molecular fraction in both is significantly lower on average than in the Galaxy. Tumlinson et al. attribute this to two effects: an enhanced UV radiation field in the Clouds, which results in a higher photodissociation rate; and a reduced $\mathrm{H}_{2}$ formation rate due to the lower dust-to-gas ratio in the metal-deficient Clouds, which reduces the grain surface area available for molecular hydrogen formation. It will be interesting, in the distant future when more sensitive far-UV spectroscopy is possible, to see how the hydrogen molecular fraction depends on metallicities in other galaxies. Tumlinson et al. found gas kinetic temperatures based on the $N(J=1) / N(J=0)$ ratio to be similar to those found for diffuse sightlines in the Galaxy. 


\subsection{2. $U V$ Observations of $H D$}

Detections of the deuterated form of molecular hydrogen were made by Copernicus (Spitzer et al. 1973; Morton 1975), and extended with FUSE observations (Ferlet et al. 2000; Lacour et al. 2005). The ratio of $N(\mathrm{HD}) / 2 N\left(\mathrm{H}_{2}\right)$ is typically around $10^{-6}$, about an order of magnitude lower than the atomic D/H ratio derived from Copernicus and FUSE observations. While the atomic $\mathrm{D} / \mathrm{H}$ ratio is an important cosmological indicator related to early big bang nucleosynthesis, it is not clear that the ratio of $\mathrm{HD}$ to $\mathrm{H}_{2}$ provides useful information constraining $\mathrm{D} / \mathrm{H}$. The main problem is that $\mathrm{H}_{2}$ is self-shielded in diffuse molecular clouds to a much greater extent than is HD, so that HD is selectively destroyed by photodissociation. Lacour et al. (2005) argue that in sufficiently dense diffuse clouds, where the molecular fraction approaches one, then the ratio of $\mathrm{HD}$ to $\mathrm{H}_{2}$ would be a valid tracer of the atomic $\mathrm{D} / \mathrm{H}$ ratio. But to date no such cloud has been observed.

\subsubsection{UV Observations of Carbon Monoxide}

Unlike $\mathrm{H}_{2}$, $\mathrm{CO}$ has allowed transitions between rotational and vibrational states as well as electronic transitions. Thus CO is widely observed through its mm-wave emission, which is collisionally excited in dense clouds; mm-wave absorption where there is a continuum radio source in the background; and infrared emission and absorption. In most cases these rotational and vibrational transitions are observed in dense clouds, but there is the possibility of obtaining such data for the same diffuse clouds that are observed in ultraviolet absorption.

Because $\mathrm{CO}$ has allowed mm-wave transitions and $\mathrm{H}_{2}$ does not, $\mathrm{CO}$ has been widely used as a surrogate for molecular hydrogen in estimating the masses of interstellar molecular clouds. But for the most part the correlation between $\mathrm{CO}$ and $\mathrm{H}_{2}$ is derived indirectly through intermediate assumed correlations between $\mathrm{H}_{2}, \mathrm{CO}$, and dust extinction, a method that is fraught with uncertainties. So there is a premium on obtaining direct measurements of both $\mathrm{CO}$ and $\mathrm{H}_{2}$ in the same lines of sight. An opportunity to do this is offered by diffuse cloud lines of sight where ultraviolet observations of both $\mathrm{CO}$ and $\mathrm{H}_{2}$ are possible. There are complications in the interpretation, though, because these are likely to be clouds with sufficient column density for $\mathrm{H}_{2}$ to be self shielding, while $\mathrm{CO}$ will not be, at least not to the same extent. Therefore the $\mathrm{CO} / \mathrm{H}_{2}$ ratio will be artificially low and a correction for $\mathrm{H}_{2}$ self-shielding must be applied.

The first UV detection of interstellar CO was achieved with a rocket-borne spectrometer flown by Smith \& Stecher (1971), who detected CO toward $\zeta$ Oph at a level of about $10^{-5}$ of the total hydrogen column density. A few Copernicus-based detections followed (Jenkins et al. 1973; Morton 1975; Snow 1975), but given the modest spectral resolving power of that instrument, it was difficult to disentangle the rotational line structure in order to derive unambiguous column densities.

Much greater success has been achieved with the high-resolution UV spectrographs such as the GHRS and the STIS aboard the Hubble Space Telescope. With spectral resolving power of about 100,000, these instruments provided UV spectra of CO bands in which the rotational lines are well resolved (e.g., Lambert et al. 1994). While the allowed electronic transitions of $\mathrm{CO}$ are typically saturated, leading to ambiguities in deriving column densities, there are a number of inter-system bands with smaller $f$-values, and from these it is possible to obtain very accurate CO column densities - though these became useful only after $f$-values had been determined through a series of laboratory and empirical astronomical studies (e.g., Sheffer et al. 2002; Eidelsberg \& Rostas 2004). Sheffer et al., taking advantage of a little-used very small entrance slit in the STIS that provides a resolving power over 200,000, found a $\mathrm{CO} / \mathrm{H}_{2}$ ratio toward $\mathrm{X}$ Per of $5 \times 10^{-6}$. 
This can be considered a lower limit to the $\mathrm{CO} / \mathrm{H}_{2}$ ratio that might be found in dense clouds, due to the selective self-shielding effect mentioned above.

Through UV spectra of $\mathrm{CO}$ it is possible to measure isotopic variations such as ${ }^{13} \mathrm{CO}$, $\mathrm{C}^{17} \mathrm{O}$, and $\mathrm{C}^{18} \mathrm{O}$, which are indicators of interest for galactic nucleosynthesis studies. While the isotopically substituted species usually have sufficient wavelength shifts to be readily distinguished from the more common form ${ }^{12} \mathrm{C}^{16} \mathrm{O}$, there are complications, most notably the fact that the isopotologues are usually less saturated and less selfshielding than $\mathrm{CO}$. In general the ${ }^{12} \mathrm{CO} /{ }^{13} \mathrm{CO}$ ratio is consistent with a lower galactic ratio of ${ }^{12} \mathrm{C} /{ }^{13} \mathrm{C}$ than the terrestrial ratio of about 90 . The isotopic ratios $\mathrm{C}^{17} \mathrm{O} / \mathrm{C}^{16} \mathrm{O}$ and $\mathrm{C}^{18} \mathrm{O} / \mathrm{C}^{16} \mathrm{O}$ can also be derived from UV carbon monoxide observations, yielding far higher dominance for $\mathrm{C}^{16} \mathrm{O}$ than is observed on Earth or in atomic interstellar gas (Sheffer et al. 2002). Sheffer et al. explain this as due to the favored photodestruction of $\mathrm{C}^{17} \mathrm{O}$ and $\mathrm{C}^{18} \mathrm{O}$ relative to $\mathrm{C}^{16} \mathrm{O}$, which is self-shielded in moderately dense clouds.

\subsection{Other Molecules Detected through UV Spectroscopy}

Many molecules have electronic transitions in the ultraviolet, but only a few have been detected in interstellar clouds - largely due to the opacity of the dust, as noted earlier in this review. Here we describe results on the small number of species that have been detected. For a list of upper limits for diffuse clouds (including species whose most favorable transitions lie at wavelengths longward of the ultraviolet) see Snow \& McCall (2006). Here we confine ourselves to UV detections only.

\subsubsection{UV Studies of Interstellar $\mathrm{OH}$}

The first molecule detected through radio observations, the $\mathrm{OH}$ radical, has electronic transitions at $1222 \AA$ and $3078 \AA$. The far-UV line was detected by Snow (1976) using the Copernicus satellite, while the near-UV line was first detected from the ground, with great effort due to atmospheric ozone interference, in the same year by Crutcher and Watson (1976) and later by Chaffee and Lutz (1977) and Felenbok and Roueff (1996). The near-UV OH features are more easily observed from space, though the transition lies near the long-wavelength cut-off for instruments such as the $I U E$ and the $H S T$, and was not covered at all by Copernicus or FUSE. While OH $\lambda 3078$ feature was readily detectable with the GHRS and the STIS, only a few observations have been reported (e.g., Snow et al. 1994). Microwave absorption due to $\mathrm{OH}$ in diffuse sightlines was observed by several authors, as summarized by Crutcher (1979) and Liszt \& Lucas (1996). Based on both the optical and the radio observations, the column densities of $\mathrm{OH}$, at the level of $10^{-8}$ of the total hydrogen, are generally consistent with gas-phase chemistry models for diffuse clouds (e.g., Black \& Dalgarno 1977; van Dishoeck \& Black 1986).

\subsubsection{UV Observations of Diatomic Carbon}

It is noteworthy that diatomic carbon $\left(\mathrm{C}_{2}\right)$ has a prominent band near $2313 \AA$, which is potentially a useful tool for measuring and analyzing this molecule. But this transition has been little exploited, largely because there are several useful transitions of $\mathrm{C}_{2}$ in the far red, which can be observed from the ground. The first attempt to detect $\mathrm{C}_{2}$ through its UV band (Snow 1978) was marginally successful, but at about the same time the utility of using the red bands became obvious (e.g., Chaffee \& Lutz 1978). A recent GHRS/HST observation of the UV band toward X Persei has produced a beautiful spectrum (Welty et al., in preparation) but in general the far-red bands are used instead for this molecule. Hence we do not include $\mathrm{C}_{2}$ in Table 1 . 


\subsubsection{UV Observations of $\mathrm{N}_{2}$ in Diffuse Clouds}

Another diatomic not expected to be abundant according to gas-phase models is $\mathrm{N}_{2}$, which has electronic transitions only in the far-UV. An early search based on Copernicus data proved negative (Lutz et al. 1979), but recently Knauth et al. (2004) have claimed a detection toward HD 124314 and have reported an additional detection toward 20 Aquilae (Knauth 2005; private communication), based on FUSE spectra in both cases. Because of blending with interstellar $\mathrm{H}_{2}$ bands as well as a prominent stellar photospheric line, and with additional concern about the possibility of telluric $\mathrm{N}_{2}$ contamination, it is very difficult to conclude unambiguously that interstellar $\mathrm{N}_{2}$ has been detected. But, as discussed by Snow (2004), Knauth et al. have addressed these issues and make a convincing case for detection. The inferred column density of $\mathrm{N}_{2}$ in the sightlines where the detections are claimed is higher than expected from gas-phase models for diffuse clouds, but lower than expected for dense molecular clouds, suggesting that dust grain surface reactions may be responsible. In view of the observational complexities, clearly it is important to search for $\mathrm{N}_{2}$ in additional lines of sight, and Knauth and colleagues are doing so, again using FUSE.

\subsubsection{Hydrogen Chloride in Diffuse Clouds}

Jura (1974), followed by Dalgarno et al. (1974), realized that ionized chlorine can undergo rapid reactions with $\mathrm{H}_{2}$ to form $\mathrm{HCl}^{+}$, which then leads to $\mathrm{H}_{2} \mathrm{Cl}^{+}$and $\mathrm{HCl}$. Jura predicted that $\mathrm{HCl}$ might be detectable through an electronic transition at $1291 \AA$, but this line was not found in a subsequent search using Copernicus data (Jura \& York 1978). The idea lay dormant for some time, until Federman et al. (1995), using HST/HST spectra, detected $\mathrm{HCl}$ (along with vibrationally excited $\mathrm{H}_{2}$ ) absorption toward $\zeta \mathrm{Oph}$. The detected column density was consistent with the predictions of models.

\subsection{Molecules on the Edge of Detection?}

Chemical models call for significant abundances of a few species that have not yet been detected, but for which ultraviolet observations may offer the best hope. These include water, hydrogen fluoride, and complex organic species such as those widely thought to be responsible for the diffuse interstellar bands.

\subsubsection{Water in Diffuse Interstellar Clouds}

Models of diffuse cloud chemistry (e.g., van Dishoeck \& Black 1986) predict an abundance of $\mathrm{H}_{2} \mathrm{O}$ of around $10^{-8}$ of the total hydrogen abundance. Water has a moderately strong UV transition near $1240 \AA$, and a few searches have been conducted, without success so far. Snow (1975) and then Smith \& Snow (1978) used Copernicus spectra to search for $\mathrm{H}_{2} \mathrm{O}$, and more recently, using STIS/HST data, Spaans et al. (1998) conducted an intensive search for the $1240 \AA$ transition of $\mathrm{H}_{2} \mathrm{O}$ toward HD 154368, placing a limit on the column density of $9 \times 10^{-12} \mathrm{~cm}^{-2}$, or about $10^{-8}$ times the total hydrogen column density, which is more stringent than the Copernicus limit for $\zeta$ Oph.

The observational limit for $\mathrm{H}_{2} \mathrm{O}$ is very close to the value predicted by the models, so we can expect that either $\mathrm{H}_{2} \mathrm{O}$ will be detected by slightly more sensitive searches, or that the models are in error. Unfortunately, due to the current hiatus in UV astronomy, it is not clear when such a search will be conducted.

\subsubsection{Interstellar Hydrogen Fluoride}

Fluorine is among the most reactive of all the common elements (e.g., atomic fluorine is the only neutral atom that undergoes an exothermic reaction with $\mathrm{H}_{2}$ ), and it has been shown that most of the fluorine in dense clouds - and likely in diffuse clouds as 
well - should be in the form of HF (Neufeld et al. 2005). Given the cosmic abundance of fluorine, this suggests that HF should be readily detectable, even in diffuse clouds.

There are some far-UV transitions of HF, the strongest lying near $951 \AA$. An intensive search for this feature has been conducted (Sonnentrucker \& Neufeld, private communication), but interference by $\mathrm{H}_{2}$ bands makes detection difficult. Currently the upper limit on $\mathrm{HF}$ is close to, but not in conflict with, the model predictions. Additional searches of the FUSE archive, for stars with varying $\mathrm{H}_{2}$ columns, is probably worth the effort.

\subsubsection{Interstellar Organic Molecules in Diffuse Clouds}

By now it is apparent that there is a large population of complex molecules permeating the diffuse interstellar medium, which manifest themselves by virtue of their infrared vibrational emission in excited regions and by the optical diffuse interstellar absorption bands (for a recent review of the evidence for large organics in diffuse clouds, see Snow \& McCall 2006).

Among the leading candidates for these large organic molecules in space are polycyclic aromatic hydrocarbons (PAHs), which consist of interlocking carbon hexagons with external attached hydrogen atoms (for a comprehensive overview, see Allamandola et al. 1992). Estimates of the total abundance of these molecules, which have an enormous variety of specific forms, are in the vicinity of $10^{-7}$ times the total hydrogen column density. Thus these species, most of which have electronic transition at ultraviolet wavelengths, might be detectable through direct absorption measurements.

Searches for UV absorption by PAHs are hampered, as usual, by the dust opacity in the $\mathrm{UV}$, and by the complexity of stellar photospheric spectra, which is especially problematic when trying to detect bands that might be broader then typical atomic interstellar lines. The most sensitive search to date, based on STIS/HST spectra, revealed no detections (Clayton et al. 2003) - but plans are afoot to do a better job, when and if the Cosmic Origins Spectrograph (COS) is installed aboard the HST (for comments on this, see $\S 5$ ).

\section{Future Prospects}

Unfortunately, the future prospects for improved UV observations of molecules are very dim at this time. The two high-resolution spectrographs on the HST (i.e, the GHRS and the STIS) have been removed or have died; the FUSE observatory is limping due to pointing problems and probably will not last much longer; and the only planned new UV instrument, the Cosmic Origins Spectrograph (COS; Green 2000), is awaiting a launch that may never occur. Worse, there is no UV instrument even in the planning stages, except for small rocket payloads that can provide only limited data.

So it is difficult to discuss future prospects for UV observations of interstellar molecules. The best hope for the near future is that the Space Shuttle will be returned to service and that the long-awaited Hubble Servicing Mission 4 will be flown. Currently the future of the Shuttle program is uncertain, pending a successful and accident-free launch in the spring of 2006. If this goes as planned, then we can realistically hope for deployment of the COS by the end of 2007 .

If and when the COS is installed and operating aboard the HST, there will be an unprecedented opportunity to conduct sensitive searches for the electronic transitions of large organic molecules thought to be responsible for the infrared emission features and possibly also the unidentified optical diffuse interstellar bands. The COS may also help in the search for water in diffuse clouds, though with only marginal spectral resolution. Because molecular observations are a high priority within the COS Science Team, we can be confident that such observations will be pursued, when and if the COS is deployed. 


\section{Acknowledgements}

We gratefully acknowledge NASA grants NAG5-11487 and NG04GL34G to the University of Colorado, as well as NASA contract NAG5-12279, for support of research used in the preparation of this review. Joshua Destree was indispensable in helping prepare this manuscript for publication.

\section{References}

Allamandola, L.J., Tielens, A.G.G.M., \& Barker, J.R. 1992, Ap. J. Suppl. 71, 733

Black, J.H. \& Dalgarno, A. 1973, Ap. J. 184, L101

Black, J.H. \& Dalgarno, A. 1977, Ap. J. Suppl. 34, 405

Boissé, P., Le Petit, F., Rollinde, E. Roueff, E., Pineau des Forêts, G., Andersson, B.-G., Gry, C., \& Felenbok, P. 2005, A\& A 429, 509

Carruthers, G.R. 1970, Ap. J. 161, L81

Chaffee, F.H. \& Lutz, B.L. 1977, Ap. J. 213, 394

Chaffee, F.H. \& Lutz, B.L. 1978, Ap. J. 221, L91

Clayton, G.C., Gordon, K.D., Salama, F., Allamandola, L., Martin, P.G., Snow T.P., Whittet, D.C.B., Witt, A.N., \& Wolff, M.J. 2004, Ap. J. 592, 947

Crutcher, R.M. 1979, Ap. J. 31, L151

Crutcher, R.M. \& Watson, W.D. 1976, Ap. J. 203, L123

Dalgarno, A., de Jong, T., Oppenheimer, M., \& Black, J.H. 1974, Ap. J. 192, L37

Eidelsberg, M. \& Rostas, F. 2003, Ap. J. Suppl. 145, 89

Federman, S.R., Cardell, Jason A., van Dishoeck, E.F., Lambert, D.L., \& Black, J.H. 1995, Ap. J. 445,325

Felenbok, P. \& Roueff, E. 1996, Ap. J. 465, L57

Ferlet, R. et al. 2000, Ap. J. 583, L69

Green, J.C. 2000, Proc. Soc. Photo-Opt. Inst. 4013, 352

Hurwitz, M. 1998, Ap. J. 500, L67

Hurwitz, M. et al. 1998, Ap. J. 500, L1

Jenkins, E.B., Reale, M.A., Zucchino, P.M., \& Sofia, U.J. 1996, Ap\&SS 239, 315

Jenkins, E.B. \& Shaya, E.J. 1981, Ap. J. 231, 55

Jenkins, E.B. \& Spitzer, L. 1975, ARA\&A 13, 133

Jura, M. 1974, Ap. J. 190, 33

Jura, M. 1975a, Ap. J. 197, 575

Jura, M. 1975b, Ap. J. 197, 581

Jura, M. \& York, D.G. 1978, Ap. J. 219, 861

Knauth, D.C., Andersson, B.-G.; McCandliss, S.R., \& Moos, H.W. 2004, Nature 429, 636

Lacour, S.; André, M.K., Sonnentrucker, P., Le Petit, F., Welty, D.E., Desert, J.-M., Ferlet, R.; Roueff, E., \& York, D.G. 2005, A\&BA 430, 967

Lambert, D.L., Sheffer, Y., Gilliland, R.L., \& Federman, S.R. 1994, Ap. J. 420, 756

Liszt, H. \& Lucas, R. 1996, A\&A 314, 917

Meyer, D.M., Lauroesch, J.T., Sofia, U.J., Draine, B.T., \& Bertoldi, F. 2001, Ap. J. 553, L59

Moos, H.W. et al. 2000, Ap. J. 583, L1

Morton, D.C. 1975, Ap. J. 197, 85

Neufeld, D.A., Wolfire, M.G., \& Schilke, P. 2005, Ap. J. 628, 260

Rachford, B. 2002, Ap. J. 577, 221

Rogerson, J.B., Spitzer, L., Drake, J.F., Dressler, K., Jenkins, E.B., Morton, D.C., \& York, D.G. 1973, Ap. J. 181, L97

Savage, B.D. \& Sembach, K.R. 1996, ARA\&A 34, 279

Savage, B.D., Drake, J.F., Budich, W., \& Bohlin, R.C. 1977, Ap. J. 216, 291

Sheffer, Y., Federman, S.R., \& Lambert, D.L. 2002, Ap. J. 572, L95

Shull, J.M. \& Beckwith, S. 1982, ARA\&A 20, 163

Shull, J.M. et al. 2000, Ap. J. 538, L73

Smith, W.H. \& Snow, T.P. 1978, Ap. J. 228, 435 
Smith, A.M. \& Stecher, T.P. 1971, Ap. J. 164, L43

Snow, T.P. 1975, Ap. J. 201, L21

Snow, T.P. 1976, Ap. J. 204, L127

Snow, T.P. 1978, Ap. J. 220, L93

Snow, T.P. 1997, in Formation $E$ Evolution of Solids in Space, eds. J.M. Greenberg and A. Li (Kluwer), p. 1

Snow, T.P. 2004, Nature 429, 615

Snow, T.P. et al. 2000, Ap. J. 538, L69

Snow, T.P. \& McCall, B.J. 2006, ARA\&A, in press

Snow, T.P., Hansen, M.M., Black, J.H., van Dishoek, E.F., Crutcher, R.M., \& Lutz, B.L. 1998, Ap. J. 504, 55

Spaans, M., Neufeld, D., Lepp, S., Melnick, G.J., \& Stauffer, J. 1998, Ap. J. 503, 780

Spitzer, L. 1946, Astr. Quart. 7, 131

Spitzer, L. \& Cochran, W.D. 1973, Ap. J. 191, L127

Spitzer, L., Cochran, W.D., \& Hirshfeld, A. 1974, Ap. J. Suppl. 28, 373

Spitzer, L., Drake, J.F., Jenkins, E.B., Morton, D.C., Rogerson, J.B., \& York, D.G. 1973, Ap. J. 181, L116

Spitzer, L. \& Zabriskie, F.R. 1959, PASP 71, 412

Tumlinson, J. et al. 2002, Ap. J. 566, 857

van Dishoeck, E.F. \& Black, J.H. 1986, Ap. J. Suppl. 62, 109

van Dishoeck, E.F. \& Black, J.H. 1989, Ap. J. 340, 273

Witt, A.N., Stecher, T.P., Boroson, T.A., \& Bohlin, R.C. 1989, Ap. J. 336, L21 